ORIGINAL ARTICLE

\title{
The importance of acknowledging clinical uncertainty in the diagnosis of epilepsy and non- epileptic events
}

\author{
R Beach, R Reading
}

See end of article for authors' affiliations

....................

Correspondence to: Dr R Beach, Jenny Lind Children's Department, Norfolk and Norwich University Hospital, Colney Lane, Norwich NR4 7UY, UK; richard.beach@nnuh. nhs.uk

Accepted 20 July 2005 Published Online First 30 August 2005
Background: Failure to recognise diagnostic uncertainty between the epilepsies and non-epileptic events may be a factor in high rates of misdiagnosis.

Aims: To explore the results of acknowledging diagnostic uncertainty in a cohort of children presenting with paroxysmal events.

Methods: Children (29 days-16th birthday) with new presentations of paroxysmal disorders were ascertained through outpatients, admissions, and accident and emergency over a two year period in a district hospital with a catchment population of 500000 . Cases were classified by diagnosis at entry and 6-30 months later. A random selection of cases was independently assessed.

Results: A total of 684 cases were ascertained. Attacks were initially classified as febrile seizures $(n=212)$, acute symptomatic epileptic seizures $(n=5)$, epilepsies $(n=83)$, unclassified (possible epilepsy) $(n=90)$, isolated epileptic seizures $(n=51)$, and non-epileptic events $(n=243)$. Case review enabled reclassification of 61 of those initially unclassified-31 to an epilepsy and 27 to non-epileptic events. In 29 the final diagnosis was never clarified. These were 23 cases with confusing or absent histories and six with short lived seizure clusters. Prognosis for these 29 cases was good; $75 \%$ had been discharged. None were on long term medication. The diagnosis in the 131 cases confirmed as epilepsy was stable. Independent review of a random sample showed full concordance with one neurologist and $20 \%$ uncertainty with another.

Conclusion: In addition to definite epilepsy or non-epileptic events it is helpful to recognise a group of cases where the diagnosis is uncertain-unclassified paroxysmal events. Reassessment of these cases enables accurate diagnosis and may prevent a hasty and incorrect diagnosis of epilepsy.
E pilepsies present in many guises and must be distinguished from a plethora of other turns and attacks of childhood. The diagnosis of epilepsy is usually a clinical one and depends mainly on a carefully taken history from a good eyewitness. The electroencephalogram (EEG) may be helpful in classifying the epilepsies, but patients with epilepsies can have normal EEGs and patients without epilepsy may show electroencephalographic abnormalities. ${ }^{1}$

Sometimes the diagnosis of an epilepsy and even classification into an epilepsy syndrome is straightforward. For example, consider a 7 year old girl who presents with uncomplicated absences. There is a good account of how these interrupt her activities for a few seconds, but without motor features. Her EEG shows bursts of typical $3 \mathrm{~Hz}$ spike wave discharges during hyperventilation induced absence. The diagnosis of childhood absence epilepsy can be made with a high degree of certainty. Some non-epileptic events are also evident from the clinical presentation. A 2 year old with episodes of pallor and transient collapse with stiffening following unexpected painful stimuli can be confidently diagnosed with reflex anoxic seizures.

In other children the presentation is not straightforward. Attacks may be vague and not well observed or described. The episodes may not fit any recognisable pattern and when the diagnosis is uncertain the interictal EEG may give no diagnostic information and can be misleading. Such cases are common in clinical practice but under-represented in the literature, which often describes the epidemiology of confirmed cases of epilepsy $y^{2-5}$ or describes misdiagnoses of epilepsy in children with non-epileptic conditions..$^{6-9}$

These themes in the literature may give clinicians the impression that all attacks should be classified either as epileptic or non-epileptic. We believe that this is a factor contributing to high rates of misdiagnosis-up to $30 \%$ in some series. ${ }^{10}{ }^{11}$ In addition to cases with "definite epilepsy" or definitely not epilepsy there are those where the diagnosis is uncertain, but epilepsy is still being considered. We have prospectively investigated the diagnosis of all paroxysmal disorders presenting to a district hospital, with particular reference to those cases where the diagnosis was uncertain at presentation.

\section{PATIENTS AND METHODS}

This was a prospective observational study from 26 March 2001 to 25 March 252003.

The setting is the Norfolk and Norwich University Hospital, a large district hospital serving a population of approximately 500 000, with 80000 aged younger than 16 years. During the study period some 12600 new inpatients and outpatients were seen. The children's epilepsy service forms part of the Norwich epilepsy service. This district service has leads for adults, learning difficulties, and children, and an epilepsy nursing service. The children's service is supported by regular clinics with a regional paediatric neurologist and a regional epilepsy interest group presents a forum for case discussion. Not all patients with epilepsy are seen within the epilepsy service and other paediatricians see children with attacks of various sorts.

\section{Case definition}

Children were included if they were aged between 29 days and their 16th birthday and presenting with definite or

Abbreviations: AED, anti-epilepsy drug; EEG, electroencephalogram; $M R I$, magnetic resonance imaging 


\begin{tabular}{lr}
\hline \multicolumn{2}{l}{ Table $1 \quad$ Diagnoses of non-epileptic events } \\
\hline Type of non-epileptic event & No. \\
\hline Infantile & \\
Apnoeic and cyanotic attacks & 31 \\
Hyperexcitability & 2 \\
Sleep related & \\
Night terrors & 6 \\
Benign neonatal sleep myoclonus & 5 \\
Sleep walking & 1 \\
Narcolepsy & 1 \\
Anoxic seizures & \\
Vasovagal syncope & \\
Reflex anoxic seizures & 60 \\
Blue breath holding & 48 \\
Behavioural & 8 \\
Non-epileptic attack disorder & \\
Other episodic behaviours & 12 \\
Fever related & 25 \\
Rigors & \\
Delirium & 12 \\
Other & 1 \\
Daydream & \\
Tics & \\
Stereotypies & \\
Migraine & \\
Paroxysmal vertigo & 20 \\
Undiagnosed & 18 \\
Total & 272 \\
\hline
\end{tabular}

possible seizures, attacks, collapses, turns, apnoeas, behaviours, or other paroxysmal disorders where a neurological cause was included in the differential diagnosis.

\section{Ascertainment}

Diagnostic information on all children admitted or seen urgently within paediatrics is routinely recorded and was regularly monitored. All children's accident and emergency records were monitored using the screening already in place as part of the hospital child protection policy. Consultant colleagues referred new outpatients to the study. We are confident of near complete ascertainment from these sources. It proved harder to ascertain children whose first event occurred after admission and the incidence of symptomatic seizures in the study is probably an underestimate. Checks were made with adult neurology and the hospital patient administration system but no additional cases were discovered.

\section{Initial diagnosis and classification}

All case records were reviewed by one observer (RCB) at the time of first presentation. Demographic, diagnostic, investigative and treatment data were collected. Cases were classified as:

- Epilepsy

- Possible epilepsy

- Isolated epileptic seizures

- Non-epileptic events

- Febrile seizures

- Acute symptomatic epileptic seizures.

\section{Definitions}

- Epilepsy: a confident diagnosis of epilepsy has been made on the basis of clinical history or clinical records, sometimes with confirmatory investigations
Table 2 Results of initial and final diagnostic classification

\begin{tabular}{lrrc}
\hline Classification & Initial & Final & Change \\
\hline Epilepsy & 83 & 131 & 48 \\
Possible epilepsy & 90 & 29 & -61 \\
Isolated epileptic seizures & 51 & 32 & -19 \\
Non-epileptic events & 243 & 272 & 29 \\
Febrile seizures & 212 & 215 & 3 \\
Acute symptomatic epileptic seizures & 5 & 5 & 0 \\
Total & 684 & 684 & 0 \\
\hline & & & \\
& & & \\
\end{tabular}

- Possible epilepsy: at presentation there were some features suggestive of epilepsy, but there was insufficient evidence to make a confident diagnosis

- Non-epileptic events: attacks that could be confidently diagnosed as one of the recognised non-epileptic conditions of childhood ${ }^{12} 13$

- Febrile seizures: seizures complicating febrile illnesses in children under 6 years and over 6 months of age

- Isolated epileptic seizures: an unprovoked single epileptic seizure or cluster of epileptic seizures during a 24 hour period

- Acute symptomatic epileptic seizures: epileptic seizures complicating an acute medical illness where the cause of the seizures could be identified.

\section{Case review}

All the case notes in the epilepsy, possible epilepsy, and isolated epileptic seizure group were reviewed between 6 and 30 months from initial classification-and reclassified where necessary. One observer (RCB) carried out these reviews. Most children with "non-epileptic events" had been discharged. Case notes of those still under follow up were reviewed. There was no review of children with febrile or symptomatic epileptic seizures.

\section{Independent review}

To check the accuracy and acceptability of diagnostic classification a sample of patients defined from random number tables with epilepsy, possible epilepsy, or nonepileptic events was selected for independent review. Anonymised case reports were prepared according to a standard proforma. Two paediatric neurologists then classified these independently.

Norwich district ethics committee gave ethical approval for the study: ref LREC 200/171.

\section{RESULTS}

\section{Ascertainment, classification, and outcome}

During the two year study 684 cases were ascertained. These children accounted for $5.4 \%$ of the 12600 children presenting to the paediatric department. A wide variety of non-epileptic events were seen as described in table l. There were marked changes in classification between initial and final review (table 2). Sixteen $(31 \%)$ of 51 presenting with isolated epileptic seizures had had further epileptic seizures and were, therefore, reclassified as epilepsies. Of the 90 children initially falling into the area of diagnostic uncertainty and classified as "possible epilepsy" in this study, it was possible to reclassify $61(66 \%)$ as a result of further information from attack descriptions, natural history, or investigation results. Thirty one of these were reclassified as epilepsies (idiopathic generalised epilepsy 7, benign childhood epilepsy with centrotemporal spikes 6, childhood absence epilepsy 2, symptomatic focal epilepsy 5, unclassified focal or tonic 
Table 3 EEG and MRI scans related to initial diagnosis

\begin{tabular}{lclll}
\hline Initial diagnosis & Total & No. investigated & $\%$ & Notes \\
\hline Epilepsy & 83 & 71 & 85 & EEG (67), MRI (24) \\
Possible epilepsy & 90 & 74 & 82 & EEG (74), MRI (24) \\
Non-epileptic event & 243 & 35 & 14 & EEG (30), MRI (5) \\
\hline
\end{tabular}

clonic seizures 11). Twenty seven were reclassified as nonepileptic events of various sorts (daydreams 7 , anoxic seizures 8, undiagnosed turns 5, other 7). The diagnosis in 29 children remained uncertain. In these cases the history remained uncertain or absent in six. Seventeen had clinical presentations that were judged insufficient to make a confident diagnosis of epilepsy. Six had a cluster of seizures lasting between 2 and 60 days. These included four with tonic clonic seizures, one with absences, and one with complex partial seizures. After 6-30 months of follow up, 21 of the children with a final diagnosis of possible epilepsy had been discharged, while eight were still under review.

Once the diagnosis of epilepsy or non-epileptic events was made, only one case was reclassified. An infant initially diagnosed as benign neonatal sleep myoclonus was reclassified as infantile myoclonic epilepsy.

\section{Case management}

Data comparing the use of EEGs, magnetic resonance brain imaging (MRI) scans, and anti-epilepsy drug (AED) therapy in the various diagnostic groups are found in tables 3 and 4. Omission of the EEG in children diagnosed with epilepsies reflects children moving into the area with existing proven epilepsies and children with complex disability where epilepsy classification was considered of limited value. Children with an initial diagnosis of possible epilepsy were investigated as frequently as those with epilepsy, but the proportion treated with an AED was much lower. Children with non-epileptic events were seldom investigated in this way and only two received an AED. No child with possible epilepsy or non-epileptic events remained on an AED at the time of reclassification. EEG and MRI scans on children with non-epileptic events were all normal.

\section{Independent review}

One of the paediatric neurologists felt that 2 of the 10 epilepsy cases put up for review were uncertain and should be reclassified as possible epilepsy, while the second neurologist classified all 10 as epilepsy. This neurologist felt that 2 of the 10 cases of non-epileptic events put up for review should be reclassified as possible epilepsy while the first classified all 10 as non-epileptic events. The possible epilepsy cases reviewed produced a variety of responses but no unanimity (table 5). There was always one of the neurologists in agreement with the study diagnosis.

\section{DISCUSSION}

Even though the pathophysiologies of epilepsy and other paroxysmal disorders are usually distinct their clinical

Table 4 Anti-epilepsy drug (AED) therapy related to final diagnosis

\begin{tabular}{lccl}
\hline Final diagnosis & Total & No. receiving AED & $\%$ \\
\hline Epilepsy & 131 & 101 & 77 \\
Possible epilepsy & 29 & 4 & 13 \\
Non-epileptic event & 272 & 2 & 0.7 \\
\hline
\end{tabular}

presentations may be similar leading to difficulties in making an accurate diagnosis. We had used the term "possible epilepsy" to deal with this area of diagnostic uncertainty. Ninety cases (13\%) lay within this area at presentation. Further investigations, observations, or descriptions of the attacks and the natural history of their condition enabled an accurate diagnosis in 61. Similar numbers of these cases were reclassified as epilepsy or non-epileptic events. A variety of epilepsies and non-epileptic events presented diagnostic difficulty at presentation suggesting real diagnostic dilemma, as these cases are not simply a sub-group of epilepsy. Even after full investigation and follow up the diagnosis remained uncertain in 29 cases (4\%). These findings lead us to question our terminology used in these cases. Possible epilepsy is not really an appropriate term for a group of children when only one third are eventually diagnosed with an epilepsy. Although it is cumbersome we are persuaded that the term "unclassified paroxysmal events" is more accurate.

Acknowledging diagnostic uncertainty in this way has implications for case management. These children had similar levels of investigation to those with epilepsy (85\% compared with $82 \%$ ), but only $13 \%$ received an AED compared with $77 \%$ of those with epilepsy. Those whose paroxysmal events remained unclassified for more than six months seem to have a good prognosis-three quarters were free of attacks, not taking AEDs, and no longer being followed up in our department.

The risk of further seizures and a subsequent diagnosis of epilepsy after an isolated, unprovoked, afebrile epileptic seizure was 33\% after 6-30 months of follow up and is similar to other published reports. ${ }^{14}{ }^{15}$

The diagnosis of epilepsy once made did not change-nor did the diagnosis of non-epileptic events. Only one case initially diagnosed as a non-epileptic event was later reclassified as an epilepsy. No cases of epilepsy have been subsequently reclassified as non-epileptic events. This is in marked contrast to studies showing high levels of misdiagnosis. ${ }^{1011}$ While this might constitute inappropriate confidence in a diagnosis not always reviewed by an independent

Table 5 A comparison of the study diagnosis with a neurologist's diagnosis for the 15 cases of possible epilepsy subjected to external review

\begin{tabular}{ll}
\hline Neurologist 1 & Neurologist 2 \\
\hline Possible epilepsy & Possible epilepsy \\
Possible epilepsy & Possible epilepsy \\
Non-epileptic event & Possible epilepsy \\
Possible epilepsy & Epilepsy \\
Non-epileptic event & Possible epilepsy \\
Non-epileptic event & Possible epilepsy \\
Possible epilepsy & Epilepsy \\
Epilepsy & Possible epilepsy \\
Possible epilepsy & Possible epilepsy \\
Possible epilepsy & Possible epilepsy \\
Non-epileptic event & Possible epilepsy \\
Possible epilepsy & Possible epilepsy \\
Possible epilepsy & Epilepsy \\
Non-epileptic event & Possible epilepsy \\
Possible epilepsy & Epilepsy \\
\hline
\end{tabular}




\section{What is already known on this topic}

- The epilepsies need to be distinguished from a variety of non-epileptic events

- Misdiagnosis of epilepsy is common

specialist, cases where there is diagnostic uncertainty are discussed with members of the regional epilepsy network and the database is informed by the usual clinical review of patients.

It is a strength of this study that all cases of non-epileptic events, attacks, and seizures that presented were ascertained and accounted for. The hospital provides the only paediatric service for central Norfolk, so we are confident that we have full ascertainment of cases where epilepsy was a possible diagnosis. No attempt was made to determine the true incidence of non-epileptic events in the community and we assume that many non-epileptic attacks are diagnosed and managed in primary care. Our concern was with the diagnosis and management of those cases presenting to hospital and thus needing to be differentiated from the epilepsies. Only one in five of the attacks presenting to this hospital were eventually diagnosed as an epilepsy.

The weakness of this study is that the case review and classification were the work of a single clinician. Difficult cases were discussed at district epilepsy team meetings or with the regional neurologist at one of the regular outreach clinics. While numbers in the study were considered too high to permit complete reclassification by a paediatric neurologist, the random cases that were reassessed showed $100 \%$ agreement with epilepsy diagnosis by one neurologist and $80 \%$ agreement by the other.

Both neurologists and paediatricians found some cases difficult to classify as epilepsy or non-epileptic events and found it helpful to have a term to express this difficulty. There are no cases where neurologists both disagree with study diagnosis and no cases where neurologists were convinced the study diagnosis was wrong. This variation in opinion lends further support to the thesis that it is necessary to acknowledge an area where there is diagnostic uncertainty. The Dutch Study of Epilepsy in Childhood has also reported cases where there is diagnostic uncertainty-again paediatric neurologists were not always unanimous in their diagnosis. ${ }^{16}$ That study also advocates a conservative approach in children with paroxysmal disorders of uncertain nature. ${ }^{17}$

Our terminology of childhood attack disorders requires clarification. The epilepsies can be described using the multi axial classification of the International League Against Epilepsy task force. ${ }^{18}$ The term non-epileptic event ${ }^{8}$ describes the clinical area sometimes called "funny turns" and includes a wide variety of recognisable clinical entities. ${ }^{12}{ }^{13}$ We have used this in preference to "turns" or "funny turns" which might be perceived as trivialising the patients' concerns. Most parents and children presenting with attacks are worried these might be manifestations of a serious underlying disorder. The use of a specific term acknowledges that professionals share this concern while making it clear that exact diagnosis remains uncertain. The term "unclassified paroxysmal events" has the virtue of accuracy. The exact terminology is less important than the principle. Clinical uncertainty in the diagnosis of epilepsies and non-epileptic events must be acknowledged. Careful and repeated attention to history taking and where necessary, investigation will usually allow accurate diagnosis. For those few cases that cannot be clarified, most will resolve spontaneously. The truly

\section{What this study adds}

- Non-epileptic events are twice as common as the epilepsies as they present to a district hospital and diagnosis is uncertain in $20 \%$ of cases at presentation

- Acknowledging uncertainty-labelling such cases "unclassified paroxysmal events" - has clinical utility and may reduce misdiagnosis by reducing pressure to make a hasty but unfounded diagnosis of epilepsy

intractable or those with significant symptoms should be referred to tertiary care.

We believe a term such as unclassified paroxysmal events should have clinical utility. It is honest in describing to parents, children, and other professionals a degree of clinical uncertainty. It removes pressure to make a hasty, possibly incorrect, and stigmatising diagnosis of an epilepsy. It emphasises the importance of observing the natural history in the diagnosis of paroxysmal disorders. Acknowledging diagnostic uncertainty should not be regarded as a failure of clinical acumen, but as an acknowledgement of clinical realities.

\section{ACKNOWLEDGEMENTS}

Thanks are extended to Chris Verity and Alasdair Parker for offering advice, encouragement, and carrying out the independent assessments.

\section{Authors' affiliations}

R Beach, R Reading, Norfolk \& Norwich University Hospital, Norwich, UK

Competing interests: RCB received a grant of $£ 1000$ from Novartis Pharmaceuticals to fund database development.

\section{REFERENCES}

1 Fowle AJ, Binnie CD. Uses and abuses of the EEG in epilepsy. Epilepsia 2000;41(suppl.3):S10-18.

2 Camfield CS, Camfield PR, Veugelers PJ. Death in children with epilepsy: a population-based study. Lancet 2002;359:1891-5.

3 Kurtz Z, Tookey P, Ross E. Epilepsy in young people: 23 year follow up of the British national child development study. BMJ 1998;316:339-42.

4 Cowan LD, Bodensteiner JB, Leviton A, et al. Prevalence of the epilepsies in children and adolescents. Epilepsia 1989;30:94-106.

5 Hauser WA. The prevalence and incidence of convulsive disorders in children. Epilepsia 1994;35(suppl 2):S1-6.

6 Gibbs J, Appleton RE. False diagnosis of epilepsy in children. Seizure 1992;1:15-18.

7 Oto M, Russell AJC, McGonigal A, et al. Misdiagnosis of epilepsy in patients prescribed anticonvulsant drugs for other reasons. BMJ 2003;326:326-7.

8 Metrick ME, Ritter FJ, Gates JR, et al. Nonepileptic events in childhood. Epilepsia 1991;32:322-8.

9 Jeavons PM. Non-epileptic attacks in childhood. In: Rose FC, eds. Research progress in epilepsy. London: Pitman, 1983:224-30.

10 Chadwick D, Smith D. The misdiagnosis of epilepsy. BMJ 2002;324:495-6.

11 Chinthapalli RN. Who should take care of children with epilepsy? BMJ 2003;327:1413.

12 Stephenson JBP. Fits and faints. London: MacKeith Press, 1990.

13 Daley HM, Appleton RE. Fits, faints and funny turns. Current Paediatrics 2000;10:22-7.

14 Stroink H, Brouwer OF, Arts WF, et al. The first unprovoked, untreated seizure in childhood: a hospital based study of the accuracy of the diagnosis, rate of recurrence, and long term outcome after recurrence. J Neurol Neurosurg Psychiatry 1998;64:595-600.

15 Shinnar S, Berg AT, Moshe SL, et al. The risk of seizure recurrence after a first unprovoked afebrile seizure in childhood: an extended follow up. Pediatrics 1996;98:216-25.

16 Stroink H, van Donselaar CA, Geerts AT, et al. The Dutch study of epilepsy in childhood. Interrater agreement of the diagnosis and classification of the first seizure in childhood. $J$ Neurol Neurosurg Psychiatry 2004;75:241-5.

17 Stroink H, van Donselaar CA, Geerts AT, et al. The accuracy of the diagnosis of paroxysmal events in children. Neurology 2003;60:979-82.

18 Engel J. A proposed diagnostic scheme for people with epileptic seizures and with epilepsy: report of the ILAE task force on classification and terminology. Epilepsia 2001;42:796-803. 